\section{P269 ACQUISITION OF EPIDEMIC PSEUDOMONAS AERUGINOSA STRAINS IN NON-CF BRONCHIECTASIS PATIENTS}

${ }^{1} \mathrm{C}$ Keil, ${ }^{2} \mathrm{~S}$ Manzoor, ${ }^{1} \mathrm{~S}$ Gossain, ${ }^{3} \mathrm{~K}$ Hardy, ${ }^{2} \mathrm{JL}$ Whitehouse. ${ }^{1}$ Public Health England Birmingham Laboratory, Birmingham, UK; ${ }^{2}$ Heart of England Foundation NHS Trust, Birmingham, UK; ${ }^{3}$ Institute of Microbiology and Infection, University of Birmingham, Birmingham, UK

\subsection{6/thoraxjnl-2016-209333.412}

Background Pseudomonas aeruginosa is an opportunistic pathogen that chronically colonises the lungs of bronchiectasis patients and is associated with a decline in lung function. Epidemic strains of $P$. Aeruginosa including Liverpool, Manchester and Midlands1 , have been described in cystic fibrosis (CF) patients and have been associated with increased morbidity and mortality, but have not previously been described in broncheiectasis patients. This study aimed to establish if there was cross transmission of $P$. aeruginosa amongst bronciectaisis patients and if epidemic strains were present.

Material/methods During the period February 2013 to May $2014 P$. aeruginosa isolates from sputum samples from bronchiectasis patients were collected. The samples were taken in both primary and secondary care. All isolates were epidemiologically typed using multiple-locus variable number tandem repeat (VNTR) typing. For 20 patients multiple isolates from the same sample were analysed and for 14 patients multiple samples were analysed. A strain-specific PCR to identify the Midlands-1 and Liverpool epidemic $P$. aeruginosa strains was used on a sub-set of isolates that had VNTR profiles related to known Liverpool and Midland-1 epidemic strains.
Results VNTR profiles were obtained for a total of 144 isolates from 84 patients. A total of 126 unique VNTR profiles were observed. Typing of multiple isolates from the same sample and the same patient revealed multiple types, with STRD $>3$ in $10 / 20$ isolates from the same sample and $14 / 15$ of multiple samples from the same patient. A total of 3 patients were chronically infected with epidemic strains, one with Midlands- 1 and two with Liverpool. All of these patients had had long stays on the cystic fibrosis ward where patients with the epidemic strains are nursed.

Conclusions Cross transmission of the $P$. aeruginosa epidemic strains from the CF population to the bronchiectasis patients occurred on several occasions. However, there is no evidence of cross transmission of non-epidemic strains amongst the bronchiectasis population. This study provides evidence for segregating bronchiectasis and cystic fibrosis patients to prevent acquisition of epidemic strains. Prospective data is required to see if patients develop Pseudomonas aeruginosa infection if seen in non-segregated clinic spaces.

\section{P270 STENOTROPHOMONAS MALTOPHILIA INFECTION IN A BRONCHIECTASIS COHORT}

${ }^{1}$ E Pigott, ${ }^{2} S$ Cowman, ${ }^{2} \mathrm{R}$ Wilson, ${ }^{2} \mathrm{MR}$ Loebinger. ${ }^{1} S t$ George's, University of London, London, UK; ${ }^{2}$ Royal Brompton and Harefield NHS Foundation Trust, London, UK

10.1136/thoraxjnl-2016-209333.413

Stenotrophomonas maltophilia has uncertain clinical significance in bronchiectasis patients. This retrospective study describes the

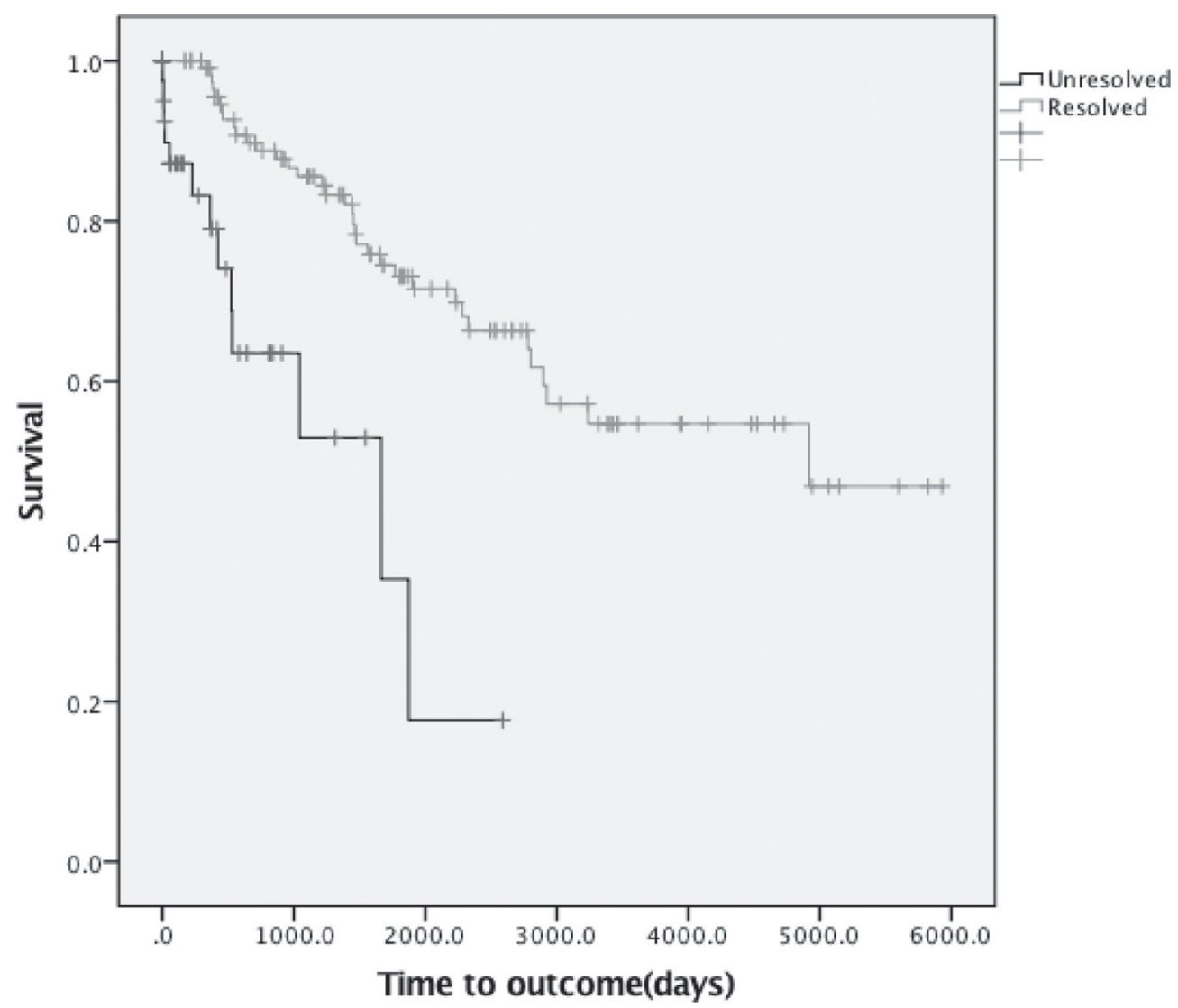

Abstract P270 Figure 1 Kaplan-Meier plot illustrating the survival of bronchiectasis patients, with S. maltophila infection resolved (grey) and unresolved (black) subgroups. There is a statistically significant difference between the plots (log rank test; $p<0.001$ ). 